\title{
Polysèmes
}

Revue d'études intertextuelles et intermédiales

\section{L'un et le multiple ou la question de l'intempestivité dans les triptyques de Francis Bacon}

Unicity and Multiplicity: The Question of Untimely Art in Francis Bacon's

Triptychs

\section{Séverine Letalleur-Sommer}

\section{OpenEdition}

\section{Journals}

\section{Édition électronique}

URL : http://journals.openedition.org/polysemes/1811

DOI : $10.4000 /$ polysemes. 1811

ISSN : 2496-4212

Éditeur

SAIT

Référence électronique

Séverine Letalleur-Sommer, « L'un et le multiple ou la question de l'intempestivité dans les triptyques de Francis Bacon », Polysèmes [En ligne], 17 | 2017, mis en ligne le 30 avril 2017, consulté le 20 avril 2019. URL : http://journals.openedition.org/polysemes/1811 ; DOI : 10.4000/polysemes. 1811

Ce document a été généré automatiquement le 20 avril 2019.

Polysèmes 


\title{
L'un et le multiple ou la question de l'intempestivité dans les triptyques de Francis Bacon
}

\author{
Unicity and Multiplicity: The Question of Untimely Art in Francis Bacon's
}

Triptychs

Séverine Letalleur-Sommer

"I always think of myself not so much as a painter but as a medium for accident and change."1

1 Le thème de l'intempestivité s'inscrit au cœur de la pratique artistique de Francis Bacon qui évoquait souvent l'importance accordée au hasard et à l'accidentel dans l'élaboration de ses toiles ${ }^{2}$. Il faisait allusion à quelque chose qui allait à l'encontre d'une réalisation programmatique et d'une élaboration attendue et réfléchie de la forme. De fait, il arrivait toujours un moment où l'artiste, laissant libre cours à son inspiration, infligeait à la toile le coup de brosse, de balayette ou d'éponge aléatoire qui scellerait le destin de l'ensemble (postérité ou destruction) : «jamais vous ne finiriez un tableau en jetant soudain quelque chose dessus ou bien feriez-vous cela? [sic] - Oh oui! Dans ce triptyque récent, sur l'épaule du personnage qui vomit dans le lavabo, il y a comme un coup de fouet de peinture blanche qui s'en va comme ça. Et bien je l'ai fait au tout dernier moment et je l'ai simplement laissé $»^{3}$. Telle une signature griffonnée au bas de la toile.

Ces traces intempestives, qui relèvent de l'inattendu, confèrent dynamisme et vitalité au tableau car, au lieu de figer la forme, elles la dynamisent en la déformant, elles l'animent. Ainsi, à propos de l'utilisation du chiffon ou de la brosse en chiendent, Bacon évoque le lieu de la première marque, qu'il cherche à réitérer car il est selon lui un point de vitalité ${ }^{4}$ . Les coups de chiffon et autres traces d'éponge, s'ils brouillent les traits d'un portrait ou brisent les contours d'une silhouette, permettent aussi l'émergence de la Figure au sens deleuzien du terme - c'est-à-dire l'émergence d'une forme proprement picturale bien plus suggestive que la simple illustration figurative. Pourquoi ? Parce que précisément le peintre exploite les manifestations spontanées de la vie, sources et expressions de 
sensations, afin de « les [réinjecter] dans un ensemble visuel » (Deleuze 92). Plus loin, l'on pourra se demander s'il n'en va pas de même de l'historien de l'art qui s'efforce de relier entre elles des formes éclectiques, survenant parfois à contretemps ou de façon inopinée, afin de les replacer dans un ensemble temporel cohérent.

\section{Retranscription atemporelle d'un chaos vital}

3 Les giclées de peinture projetées au dernier moment sur la toile coagulent ${ }^{5}$ un moment de vie paroxystique. Elles sont une façon pour le peintre d'agir "contre le temps " pour reprendre la formule de Nietzsche (94). Elles incarnent cet instant critique où règne la plus grande incertitude quant à la destinée de l'œuvre puisque, selon le résultat obtenu, Bacon pouvait parfaitement décider que le tableau ne valait plus rien et le détruire, ou au contraire considérer qu'il était achevé. On sait le goût que l'artiste nourrissait pour le jeu, la roulette notamment, et l'on retrouve un peu de ce tirage au sort dans sa pratique artistique (Bacon \& Sylvester 104-105).

4 Ces actions spontanées, génératrices de formes ou au contraire iconoclastes, s'inscrivent cependant dans un contexte où prévalent rigueur et maîtrise:cadres, arêtes de parallélépipèdes enfermant les chairs exposées et paradoxalement parfois déliquescentes, cercles-loupes concentrant le regard sur tel ou tel point du tableau, scénographie symétrique, flèches rotatives orientant l'observation. On assiste à la mise en place d'un dispositif optique dont on a l'impression qu'il est exclusivement dévolu à l'observation et à l'exposition de figures qui, elles, suggèrent au contraire une béance, une dislocation voire une décomposition de la forme. Comme le dit Bacon lui-même: «I believe in a deeply ordered chaos $»^{6}$. Or le format triptyque ajoute au sentiment de désordre contenu et semble partie intégrante de ce dispositif d'ordonnancement implacable. Il suggère la volonté réitérée de circonscrire un espace, de le maitriser et de le dupliquer afin de mieux y exhiber l'insolite, la surprise de l'apparaitre: «Il y a toujours, me semble-t-il, en peinture, et peut-être est-ce comme ça dans les autres arts, une part de maitrise et une part de surprise» (Bacon 68). Tout se passe en effet comme si l'artiste créait les conditions propices au surgissement d'un tressaillement inattendu, incalculable, intempestif en vidant littéralement l'espace de la toile ${ }^{7}$. D'ailleurs, le tressaillement est parfois lui-même évidement, comme s'il s'agissait de contenir l'inexorable liquéfaction du moi, de l'empêcher de sombrer dans le chaos et l'indiscernable.

5 La préparation, la répétition du même geste, nécessaire à l'acquisition de la maitrise artistique, inscrivent l'œuvre dans la durée, mais l'exécution corrigée du geste réussi confère sa valeur à l'instant : « Les instants sont distincts parce qu'ils sont féconds. Et ils ne sont pas féconds par la vertu des souvenirs qu'ils peuvent actualiser, mais bien par le fait que s'y ajoute une nouveauté temporelle convenablement adaptée à un progrès " (Bachelard 86). Et, de fait, il s'agit bien pour Bacon de faire place nette car « il n'y a rien en dehors de l'instant présent et l'instant présent, c'est l'instant de la perception et de la sensation $»^{8}$; peut-être aussi, l'instant, valorisé et valorisant, où il sent tout son être se condenser en un mouvement juste et précis.

6 Les instants de vie dont il est question s'incarnent tout particulièrement dans les figurations de réactions instinctives. Ainsi, « [...] l'artiste est capable d'ouvrir ou, dirai-je plutôt, de desserrer les valves de la sensation et ainsi de renvoyer plus violemment le spectateur à la vie » (Bacon \& Sylvester 46). On notera à ce propos la récurrence du motif $\mathrm{du}$ cri, expression directe et instinctive d'une douleur physique ou morale ${ }^{9}$. On pourra 
alors émettre l'hypothèse que le cri incarne l'émission vocale signalant, d'une part, la possibilité pour le spectateur d'être hélé par la forme sur l'instant, mais aussi d'en être expulsé immédiatement par le biais d'un mouvement d'éjection à l'avant du cadre; comme une invitation à ne pas rester trop longtemps devant le tableau, en saisir simplement l'immédiateté sensorielle, nerveuse ${ }^{10}$ sans en intellectualiser la réception. Le cri est aussi une façon de suggérer l'évidement nécessaire à la mise en valeur d'une unité perceptive forte, motif de l'évidement que l'on retrouve dans le tuyau d'évier ou le siphon de la cuvette des toilettes, dans les vomissements et les éviscérations ou encore, sur un autre mode, dans la présence d'interrupteurs ou de rideaux qu'il suffit de tirer pour plonger le tableau dans la plus profonde obscurité et ainsi mettre un terme au supplice. Dans le même ordre d'idées, Bacon nous offre aussi la possibilité de pénétrer dans l'envers épidermique de la toile ${ }^{11}$ - il peignait toujours du «mauvais » côté de la toile ${ }^{12}$, inversant le dedans et le dehors - là où se loge la sensation, le système nerveux. On retrouve le motif de la pénétration dans la piqûre, sensation exacerbée, dans la présence de la seringue hypodermique (Nu couché avec une seringue hypodermique, 1963) ${ }^{13}$.

On le voit, ces micro-événements infra-picturaux ne sont pas sans répercussion sur le plan de la réception hors cadre. Ainsi l'intempestivité infra-picturale se répercute à l'extérieur du cadre chez le spectateur, pris de court et malmené par l'expérience visuelle proposée. Car c'est bien là l'objectif de Bacon : provoquer un « choc visuel » ${ }^{14}$. La violence des images peut provoquer un rejet et une aversion tout aussi violents de la part du spectateur. Ce phénomène de rejet est dû au caractère intempestif de l'apparition : on ne s'y attend pas, on ne le veut pas. Il est dû aussi au mimétisme et à l'empathie qui accompagnent tout phénomène de perception : « une manière de saisir les intentions et/ ou émotions d'autrui consiste à faire soi-même l'expérience des états physiques d'autrui » (Andrieu 15). Ceci trouve une illustration dans le fait que, comme le fait remarquer Didier Anzieu, Bacon recouvrait ses tableaux d'une plaque de verre, enveloppe froide et transparente, seconde peau permettant de protéger la surface comme écorchée du tableau, mais aussi une façon de substituer à la représentation le reflet du spectateur dont il s'agit d'éveiller la conscience, d'obtenir la reconnaissance ${ }^{15}$. Pour Jean-Pierre Changeux, l'artiste cherche par son œuvre à « retrouver les autres hommes » par l'empathie. Ainsi, l'« "inhibiteur de violence" [est] un dispositif inné destiné à faire cesser la violence de l'agresseur par des signes de souffrance, de détresse, pleurs, cris... qui entraînent un arrêt de l'agression et font appel à la compassion » (Changeux 138-139). Peut-être s'agit-il en effet là aussi de désarmer le spectateur.

8 Par la suite, le temps fait son œuvre et les formes deviennent familières, la sensation s'émousse. Un tel phénomène a été récemment exploré dans le domaine des neurosciences sur le plan de la réception auditive à propos du Sacre du Printemps de Stravinsky: «[...] the audience's rioting at the first performance of Stravinsky's Rite of Spring may be accounted for by the response of certain neurons to unfamiliar sounds [...] when these neurons fail to find a pattern in the sounds to which they are being subjected, an excessive discharge of dopamine can follow, producing something like schizophrenia " (Massey 102). Jonah Lehrer montre qu'une fois les sons nouveaux intégrés par les musiciens, ces derniers se mirent à apprécier la composition de Stravinsky : «Over time, the auditory cortex works the same way; we become better able to hear those sounds that we have heard before [...] We are built to abhor the uncertainty of newness » (Lehrer 142).

9 Ainsi, dès que la toile est offerte aux regards, elle s'extrait de son historicité propre pour pénétrer le champ de celle d'une multiplicité d'observateurs potentiels qui vont balayer, 
nettoyer, lisser, polir les aspérités originelles comme le fait parfois Bacon lorsqu'il strie la toile de lignes verticales (Étude d'après le portrait du pape Innocent X par Vélasquez, 1953). L'image vient alors réalimenter le flux de clichés auquel elle s'était péniblement arrachée, permettant l'émergence d'autre chose ou sombrant dans l'oubli. Ce moment constitue un raccord intempestif car incalculable entre le temps propre de l'artiste, dans l'antre clos et atemporel de son atelier, lieu du jaillissement de la forme, et celui d'une actualité qui lui confèrera un aspect polymorphe, démultiplié aussitôt périmé.

\section{Réinscription de l'œuvre dans le temps : différence et survivance} elle s'offre aux regards existent en amont de la production artistique. L'œuvre est traversée par des influences qui s'exercent à plus ou moins grande distance, elle est également soumise à des interprétations et expressions diverses qui en régénèrent toujours le sens.

11 À la fin de son histoire de l'art, Ernst Gombrich nous rappelle que : «[...] l'“art" n'est pas quelque chose qui existe en soi. Il n'y a que des artistes, des hommes et des femmes qui ont reçu ce don merveilleux d'équilibrer des formes et des couleurs jusqu'à ce qu'elles sonnent juste et - ceux-ci sont plus rares - qui possèdent cette intégrité de caractère qui ne peut se satisfaire de demi-solutions, qui renoncera aux effets superficiels, aux succès faciles pour leur préférer le labeur harassant d'un travail sincère » (Gombrich 597). L'art, loin d'être cet idéal transcendant la multiplicité de ses états successifs, n'existe pas en dehors de ses divers modes d'apparition, autant de surgissements ponctuels et contingents dont il s'avère difficile d'envisager la provenance comme le destin, selon un mode " successif, narratif et linéaire » (Didi-Huberman 69). C'est ainsi que Gombrich nous met en garde, d'une part, contre le public qui exigerait de l'artiste qu'il reproduise ce que «jusqu'à présent on a communément désigné par le mot art » et, d'autre part, ceux [critiques et intellectuels] qui réclament du même artiste qu'il «crée du "nouveau" ... chaque œuvre nouvelle [devant] apporter un style nouveau » (Gombrich 596). Il finit par souligner que l'existence de l'art dépend en réalité de la qualité de sa réception : « C'est à nous de veiller à ce que le fil de la tradition ne se rompe point et à ce que des possibilités restent ouvertes aux artistes d'ajouter à cette précieuse rangée de perles que le passé nous a laissée en héritage [...]. [L]es événements du jour ne se transforment en histoire qu'une fois que nous avons pris suffisamment de recul pour savoir quelle incidence ils ont sur le cours ultérieur des choses » (Gombrich 597-599). photographie, un temps de pose. Loin d'être mue par un déterminisme absolu, elle s'élabore et se réélabore longtemps après que son objet s'est manifesté, toujours $a$ posteriori et au gré de celui qui l'envisage et la pense à un moment donné. À l'historien incombe la tâche de prendre en compte un temps tel qu'il est théorisé par Carlyle : « un remous fait d'actes et de blocs (solids), innombrables et simultanés, [...] le chaos de l'étant » (Didi-Huberman 69).

13 Ainsi, sur le moment, la portée d'une œuvre reste en grande partie imprévisible de même que sa provenance; le paradoxe résidant en cela que l'événement artistique rompt avec une histoire qu'il contribue pourtant à ébaucher. Selon un modèle plus rhizomatique que 
strictement hiérarchisé, le lointain peut poindre et se substituer au plus proche chronologiquement, alors que les germes d'influences futures se font jour hic et nunc et ce, pour deux raisons. Premièrement, la représentation picturale est toujours renouvellement de la forme (aussi infime soit-il). Deuxièmement, elle est destinée à être vue donc à laisser à son tour une trace susceptible de réapparaître ailleurs à plus ou moins long terme.

Nombreuses sont les toiles de Bacon illustrant ce phénomène d'hétérochronie (DidiHuberman 69). Des pans d'histoire sont réactivés par le biais de clichés ou d'œuvres picturales ayant sédimenté au fond de sa conscience : le brassard à croix gammée associé à la cocarde tricolore piquée dans des chairs broyées rappellent la barbarie, celle d'un conflit encore frais mais aussi celle, bien antérieure, de la Terreur (Crucifixion, tryptique, 1965). Autre exemple d'images fossilisées issues d'associations hétérochroniques: la figure du pape Innocent $X$ tel qu'il fut peint par Vélasquez (Étude d'après le portrait du pape Innocent X par Vélasquez, 1953), pape dont le visage a été remplacé dans le triptyque de Bacon par celui de la nurse du Cuirassé Potemkine ${ }^{16}$, déformé par la douleur alors que cette dernière vient d'être atteinte à l'œil par un coup de sabre. Ce sont là autant d'imagesfantômes qui viennent hanter l'imaginaire baconien: «Bien sûr, j'ai été littéralement obsédé par certaines œuvres des grands peintres du passé [...] J’ai été hanté par cette œuvre, par les reproductions que j'en avais vues. C'est un portrait tellement extraordinaire » (Bacon 131). En aval, les peintures de Bacon seront reprises dans au moins deux films : Théorème ${ }^{17}$ et Le Dernier Tango à Paris ${ }^{18}$.

La notion de hantise renvoie directement au concept warburgien de Nachleben longuement analysé par Georges Didi-Huberman, c'est-à-dire de formes survivantes qui transcendent l'espace, le temps et la volonté même de l'artiste pour ressurgir lorsque l'on s'y attend le moins. Elles sont une allusion à l'impureté du temps. Comme un fleuve qui charrierait de la boue, le temps s'écoule certes de façon irréversible mais en entraînant dans son cours des éléments hétéroclites détachés, comme des blocs de pierre coincés dans des anfractuosités et soudain libérés.

En ce sens, l'image est un artefact culturel (au sens d'" artifact») extrêmement sophistiqué, colporté de-ci de-là, retravaillé et malmené, subverti au gré des circonstances. Malgré les apparences, rien n'y subsiste de naturel, ni les chairs à vif, ni les convulsions, ni les bouches révulsées, ni les visages aux traits bouffis comme couverts d'ecchymoses (Autoportrait, 1976). C'est peut-être là ce que Bacon entend signifier en affublant ses figures de contours aberrants (Leiris 34-35), une autre forme de déplacement et d'intempestivité. D'autant que le peintre est convaincu que l'art moderne, déserté par le sacré qui animait encore les œuvres de Vélasquez ou Rembrandt (Jaubert 14), est condamné au jeu, à la répétition absurde de gestes devenus insensés, seuls vestiges des splendeurs passées, un peu comme «les jeux d'enfant (arcs, frondes, crécelles, osselets ou cartes à jouer) [sont] des survivances de vieilles pratiques fort sérieuses (divination, guerre) » (Didi-Huberman 69).

17 Ruptures par la subversion, réapparitions spectrales sont autant de tentatives de raccordement à une réalité hétérogène, parcellaire, qui ne nous offre que peu de prise pour la saisir, et c'est paradoxalement par le biais de ces prélèvements, de ces intrusions singulières, de ces piqûres, pour reprendre l'image de la seringue, et autres rencontres intempestives que l'on peut, sous couvert d'une interception présente, établir un lien entre plusieurs moments et se forger une histoire complexe faite d'échos, de résonances et d'annonces. Seul élément de certitude, le temps de l'un est irréversible et conduit 
inéluctablement de la naissance à la corruption des chairs et de la matière : passé présent - futur ; un rythme ternaire que l'on retrouve dans le format triptyque et dans le chromatisme de Bacon passant du rose tendre, à un bel orange puis au violacé suggérant les lividités cadavériques.

\section{Représentation de la temporalité dans l'art de Bacon : le format triptyque}

18 La question de l'intempestif pose également le problème de l'inscription du temps dans l'art pictural ; un art dont l'immobilité semble faire osciller l'œuvre entre prélèvement immédiat de l'instant représenté et fixation du détail, comme dilaté artificiellement.

De fait, en inscrivant le temps de l'acte sur la toile par le biais du balayage, du lissage ou de l'effacement, Bacon rend la vitesse d'exécution, un geste qui ne renvoie qu'à lui-même et à sa temporalité propre. C'est une façon pour lui de révéler sa présence comme action sur le monde, encore une fois peut-être "contre le temps", car Bacon nettoie, polit ou brosse toujours un déjà-là, une forme sous-jacente : «cet acte, ou ces actes supposent qu'il y ait déjà sur la toile (comme dans la tête du peintre) des données figuratives, plus ou moins virtuelles, plus ou moins actuelles. Ce sont précisément ces données qui seront démarquées, ou bien nettoyées, balayées, chiffonnées, ou bien recouvertes, par l'acte de peindre » (Deleuze 93). Le figuratif est ainsi mêlé d'abstrait, le temps de la représentation se mue en présence et présentation ${ }^{19}$.

L'on peut par ailleurs considérer le format triptyque comme un discours métapictural sur le caractère kaléidoscopique des représentations visuelles et sur la vision en général. La vision consiste en la fragmentation et la reproduction succincte du réel, « une sténographie du réel $»^{20}$ comme le précise Bacon. Pour lui : «c'est en regardant que l'on apprend. C'est cela qu'il faut faire, regarder» (Bacon 130). Or regarder, c'est comprendre comment l'on voit, plus que ce que l'on voit. La vision, et la façon dont nous enregistrons les images visuellement, est toujours parcellaire, y compris à l'échelle rétinienne. Elle nous en apprend plus sur un fonctionnement corporel qui est notre seule façon d'être au monde, plutôt que sur le monde lui-même. Cette parcellisation indique que le lien de causalité est une représentation de l'esprit puisqu'il n'y a pas de continuité de la perception. L'absence de continuité oblige à une construction du temps artificiellement fluidifiée et logicisée a posteriori alors que, nous venons de le voir, le temps est impur tout comme le flux de la conscience ${ }^{21}$. Ceci apparaît de façon manifeste dans le Triptyque 1986-1987 où le portrait subverti du président Wilson au sortir de la conférence de Versailles, côtoie la reproduction d'un meuble de la pièce dans laquelle Trotski fut assassiné à Mexico, meuble recouvert d'un drap blanc maculé de sang. Sur le panneau central : un personnage qui se liquéfie : «Est-il possible qu'il n'y ait aucun rapport entre d'une part une image qui renvoie au traité de Versailles, dont il est bien connu qu'en imposant aux Allemands une humiliation sévère, il a fait le lit du nazisme en alimentant l'idéologie revancharde et d'autre part l'assassinat qui, à la veille de la seconde guerre mondiale, laisse les mains totalement libres à Staline ? [...] le "lien narratif" entre les panneaux est donc absolument flagrant. Ceux-ci offrent un raccourci brutal de ces décennies sanglantes qui furent aussi celles de la jeunesse du peintre » (Mavrakis).

Bien que Bacon ait cherché à supprimer l'istoria, précisément par le biais de la forme triptyque, des considérations matérielles l'ont aussi obligé à fragmenter ses œuvres (les 
panneaux ne peuvent dépasser la taille de l'encadrement de la porte de son atelier :1,98m sur $1,47 \mathrm{~m})^{22}$. Aussi absurde que cela puisse paraitre, cela nous rappelle les limites de notre vision construite à la mesure de ce que nous sommes. Il faut trancher dans le réel pour le rendre appréhendable, pour pouvoir le manipuler, comme le boucher s'applique à découper les quartiers de viande destinés à la dévoration. Il y a une dimension prédatrice dans le découpage pictural et visuel, prédation dont on trouve un écho dans les bêtes sauvages (Étude de babouin, 1953) et les charognards (sur le panneau de gauche de Trois Études de figures au pied d'une crucifixion, 1944 ; Triptyque, 1976) ${ }^{23}$.

Cette parcellisation de la vision provoque également une multiplication du réel et non seulement sa réduction (ou raccourci). Bacon, bien que grand admirateur du retable d'Issenheim (Matthias Grünewald, 1512-1516), prétendait s'être davantage inspiré pour ses triptyques des photographies anthropométriques prises par la police que des triptyques du Moyen Âge ${ }^{24}$. On a bien là une multiplication de l'un: face, profil gauche, profil droit (cf. le titre de l'ouvrage de Leiris). On sait également qu'il se servait pour ses portraits de photos d'identité de photomatons où un même format reprend un même visage selon différentes expressions. L'on sait aussi qu'il s'est largement inspiré de clichés de Muybridge sur la décomposition du mouvement, notamment de mouvements de corps difformes qui permettent d'attirer le regard sur un dynamisme atypique. Encore une fois, cette décomposition n'a rien à voir avec la réalité, comme le montre le paradoxe de Zénon, le mouvement ne peut se réduire à sa décomposition numérique. Il ne peut finalement y avoir multiplication infinie du réel. On assiste plutôt à un prélèvement fini du réel. Le peintre procède donc à une dissection de la vision, elle-même perçue comme décomposée : face, profil, dos, anamorphose, disparition, estompage, flou. On ne peut jamais tout percevoir en même temps. On ne perçoit que d'infimes parties de la réalité qu'il incombe au cerveau de reconstruire par le biais d'échanges interactifs entre l'autre et le soi, où l'enveloppe épidermique se comporte plus à la fois comme contenant et interface.

L'idée d'un échange épidermique trouve aussi une illustration dans la forme triptyque, notamment celle des retables souvent composés de trois volets, les deux volets latéraux pouvant se refermer sur le panneau central et ainsi en couvrir ou au contraire en découvrir l'image. Là encore, on retrouve l'évocation d'un mouvement matériel qui s'accompagne d'un sentiment d'enveloppement, de seconde peau où le spectateur est comme ceint par la représentation. La recréation d'un espace tridimensionnel par l'invitation au mouvement suscite un phénomène kinesthésique, ce qui accroît le sentiment de mise en présence et d'être au monde ${ }^{25}$. D'ailleurs Bacon avait pour projet de construire de "grandes sculptures montées sur rail et pouvant coulisser $»^{26}$. La présence de corps comme vissés dans le tableau, sorte de variante outrée du contraposto, de corps difformes ou vacillants, pointe également une forme de dynamisme.

L'artiste, en cherchant à fixer l'instant, nous met souvent devant le fait en cours d'accomplissement (aspect duratif). L'image brouillée de corps pris dans des postures agonistiques force la pupille à se concentrer plus longtemps qu'à la normale sur la forme ; ce qui, malgré la fixité de l'image, déclenche une impression de dynamisme et de dilatation puisque l'œil ne cesse d'être animé de micromouvements. Il arrive également que l'on colle à l'événement avec, au centre, ces corps enchevêtrés et/ou éventrés et déliquescents. Cependant, le format triptyque suggère aussi une chronologie classique: passé, présent, futur. Ainsi, la présence de témoins, placés à droite de l'œuvre, petits personnages, vieillards caricaturaux murmurant dans un combiné téléphonique (Sweeney 
Agonistes, triptyque, 1967) ou attablés à une sorte de bureau ridicule comme prêts à recueillir une déposition (Crucifixion, triptyque, 1965), nous rappellent l'essence de toute représentation : «L'homme étant, comme on sait, un animal culturel, ceci revient à dire comme dirait Lacan - qu'il n'a aucune communication directe au réel, aucun accès direct à son milieu d'émergence (le réel), mais n'en perçoit que des interprétations plus ou moins fantasmées, des substituts toujours déjà travaillés, distanciés, et qui s'interposent entre lui et ce différé, toujours reporté, repoussé, jamais effectivement présenté, i.e. jamais autre que représenté » (Bourquin 104). Rappelons à ce propos que pour Bacon «l'art rend compte, [...] c'est un reportage » (Leiris 52).

Toute représentation peut être perçue comme duplication, parfois outrée, de la réalité et en ce sens elle constitue un témoignage de ce qui s'est passé: "I want to remake in another medium the reality of an image that excites me $\aleph^{27}$. Notre mode d'accès au monde est toujours médié y compris à nous-mêmes. Ce que l'imagination dépose sur la toile, nous l'avons vu, constitue une recomposition intempestive et impure du perçu, lui-même le fruit d'un traitement corporel de notre présence au monde : passif - actif et accompli, trois moments évoqués par Deleuze (70), mais qui présentent aussi une conformité avec les trois temps de l'échange linguistique « je - tu - il»: le moi, l'autre, la perception de l'ensemble dans un troisième temps où le moi s'est fait autre (Benvéniste 202).

Dès lors, toute représentation peut être plus ou moins confusément entendue comme un aveu de culpabilité : j'ai vu, je sais, je ne suis plus le même, je ne suis plus innocent. Bacon en peignant Dyer assis sur la cuvette des toilettes en 1967 (Trois personnages dans une pièce, triptyque, 1967) lui a pour ainsi dire dicté sa fin; d'où la dimension proleptique du tableau, au sens littéraire du terme.

La forme nous surprend alors a posteriori, et là encore de façon intempestive, lorsque l'on ne peut que constater qu'elle était bel et bien la chronique d'une mort annoncée ; celle de Dyer dont le nom même porte le funeste présage. Dyer, celui qui meurt une première fois sur la toile des portraits que Bacon en a fait, puis réellement et dans la même position la veille d'un vernissage parisien en 1971, puis de nouveau dans les portraits posthumes qu'il lui consacre.

L'œuvre d'art est matrice et matricide. Une et multiple, elle permet la génération en même temps que la régénération et la destruction des formes. D’ailleurs si Bacon est le peintre de l'élan vital, il est aussi celui des chairs meurtries et des carcasses décomposées, à la fois contre le temps, hors du temps et victime du temps ${ }^{28}$.

\section{BIBLIOGRAPHIE}

\section{Sources secondaires}

Andrieu, Bernard. Le Monde corporel de la construction interactive du soi. Lausanne : L'Âge d'homme, 2010. 
Anzieu, Didier \& Michèle Monjauze. Francis Bacon ou le portrait de l'homme désespécé. Paris : Archimbaud, 1993.

Bachelard, Gaston. L'Intuition de l'instant (1931). Paris : Stock, 1993.

Bacon, Francis. Entretiens avec Michel Archimbaud. Paris : Gallimard, 1992.

Bacon, Francis \& David Sylvester. L'Art de l'impossible. Entretiens avec David Sylvester (1976). Genève : Albert Skira, 1995.

Benveniste, Émile. «L'antonyme et le pronom en français moderne » (1965). Problèmes de linguistique générale. Vol. 2. Paris : Gallimard, 1974.

Bourquin, Guy. « Les Représentations du langage : du figuratif au stylisé ». Dossier : La Théorisation de la Relation Image/Texte/Langage. Interfaces. Images, texte, langage 5 (1994).

Changeux, Jean-Pierre. Du vrai, du beau, du bien. Une nouvelle approche neuronale. Paris : Odile Jacob, 2008.

Deleuze, Gilles. Logique de la sensation. Paris : La Différence, 1981.

Didi-Huberman, Georges. L'Image survivante. Paris : Minuit, 2002.

Gombrich, E.H. Histoire de l'art (1950). Paris : Phaidon, 2001.

Hinton, David, réal. Francis Bacon. Halle : Arthaus Musik, 1985.

Jaubert, Alain, réal. Francis Bacon. Trois personnages dans une pièce. Paris : Arte \& Éditions

Montparnasse, « Palettes », 2007.

Lehrer, Jonah. Proust was a Neuroscientist. New York: Mariner, 2008.

Leiris, Michel. Francis Bacon, face et profil. Paris : Albin Michel, 2004.

Low, Adam, réal. Bacon, l'homme et l'arène [Bacon's Arena]. Paris : Arte Vidéo, 2006.

Massey, Irving. The Neural Imagination. Aesthetic and Neuroscientific Approaches to the Arts. Austin: U of Texas P, 2009.

Mavrakis, Annie. « Bacon et l'H(h)istoire ». La Figure du Monde, 13 février 2010. http:// www.anniemavrakis.fr/2010/02/13/bacon-et-lhhistoire

Nietzsche, Friedrich. Considérations inactuelles (1874). Paris : Folio, 1990.

Sacks, Oliver. « In the River of Consciousness ». The New York Review of Books 51.1 (January 15, 2004).

Wilde, Oscar. The Ballad of Reading Gaol (1897). Édition bilingue. Paris : Flammarion, « GF », 2008.

\section{Tableaux cités}

Three Studies for Figures at the Base of a Crucifixion (1944). Huile et pastel sur panneau de bois. $94 \mathrm{x}$ $74 \mathrm{~cm}$. Tate Gallery, Londres.

Study after Velázquez's Portrait of Pope Innocent X (1953). Huile sur toile. 153 x $118 \mathrm{~cm}$. Des Moines Art Center, Des Moines.

Study of a Baboon (1953). Huile sur toile. 198,3 x 137,3 cm. Museum of Modern Art, New York. Lying Figure with Hypodermic Syringe (1963). Huile sur toile. 198 x $145 \mathrm{~cm}$. Collection privée. Crucifixion (1965). Huile sur toile. 197,5 x 147 cm. Bayerische Staatsgemäldesammlungen, Munich. 
Triptych-Inspired by T.S. Eliot's Poem “Sweeney Agonistes” (1967). Huile sur toile. 198 x 147,5 cm. Hirshhorn Museum and Sculpture Garden, Smithsonian Institute, Washington.

Triptych (1973). Huile sur toile. 198 x 147,5 cm. Collection privée.

Triptych (1976). Huile sur toile. 198 x 147,5 cm. Collection privée.

\section{NOTES}

1. Adam Low, réal., Bacon, l'homme et l'arène [Bacon's Arena], Paris : Arte Vidéo, 2006, 1h6'20".

2. « [...] mon mode de travail est tout à fait accidentel » (Bacon \& Sylvester 47).

3. À propos de Triptyque, mai-juin 1973, panneau de droite (Bacon \& Sylvester 188, également cité dans Deleuze 103).

4. «The first mark that you make, where the brush makes that mark, it has a vitality, but when you've got the overall thing created it's lost », David Hinton, réal., Francis Bacon, Halle : Arthaus Musik, 1985, 20'32".

5. Bacon parle de « coagulation de marques non-représentatives » (Bacon \& Sylvester 118).

6. Hinton, 17'20". Notons à ce propos cette remarque de Michèle Monjauze : «[...] les créateurs alcooliques, ou psychotiques, se jettent sur l'espoir fou, dans la visée d'une création d'eux-mêmes en tant que sujets, de maîtriser enfin leur chaos ordinaire par l'acte créateur» (Anzieu \& Monjauze 30).

7. Deleuze montre qu'il faut toujours avant de peindre se débarrasser des clichés qui préexistent au tableau. Notons à ce propos le contraste flagrant entre le capharnaüm accumulé dans l'antre de l'artiste (atelier reconstitué à Dublin), « un tas de compost » (Alain Jaubert, réal., Francis Bacon. Trois personnages dans une pièce, Paris : Arte \& Éditions Montparnasse, «Palettes » 2007, 8') selon ses mots et le dépouillement relatif qui règne dans ses toiles (Deleuze 83-88).

8. «What is life but sensation? [...] Do you think there's anything apart from the moment. No. I believe in nothing " (Hinton, 30'55").

9. Bacon expliquait la présence de bouches en train de crier dans ses peintures par sa fascination pour les couleurs de l'intérieur de l'orifice buccal : ivoire des dents, nuances de rouges et de rose des gencives, de la muqueuse et de la langue. Or lorsque Melvyn Bragg lui fit remarquer que les bouches qu'il peignait étaient la plupart du temps toutes noires, il répondit simplement qu'elles n'étaient pas réussies : «- Francis, most of your mouths are black. - I've never been able to make the really successful mouths » (Hinton, 32'25").

10. Voir à ce propos les réflexions de Francis Bacon sur la photographie, la peinture et le système nerveux (Bacon \& Sylvester 117).

11. Voir la notion de moi-peau et d'enveloppe psychique théorisée par Didier Anzieu pour lequel les œuvres de Bacon révèlent notamment un « vacillement des oppositions constitutives [dont] le dedans-dehors pour l'axe de la contenance » (Anzieu \& Monjauze 14).

12. " the unprimed side of the canvas» (Hinton, 19'32").

13. «[...] il se peut que la seringue, évocatrice d'extases prohibées, ait surtout cette fonction: situer dans l'actualité, donc dans la réalité, un nu qui, autrement, ne serait guère moins mythologique qu'une Vénus (telles celles de Velázquez ou autres grands peintres classiques)» (Leiris 29).

14. "I like the starkness of the image. I want to give me a shock [sic]; Shock is a form of expression but what expression it is, I don't know, it's a visual shock, it's not a shock about what you could get from a story " (Hinton, 22'20").

15. «Le peintre recommande de mettre ses tableaux sous verre. Il fournit la justification suivante: "Le verre aide à l'unité du tableau. J'aime aussi la distance que le verre crée entre ce qui a été fait et le spectateur. J'aime que l'objet soit, pour ainsi dire, mis aussi loin que possible." 
[...] La mise sous verre du personnage peint que le visiteur vient regarder signifierait la rencontre avec le regard indifférent qu'une mère porte à son nourrisson et à ses cris de rage et de douleur. En regardant ce personnage, le visiteur s'y regarde et reconnaît, superposés, l'image réelle de son propre visage réfléchi par le verre et le portrait, sur la toile, d'une souffrance intérieure liée au vide de la non-reconnaissance et à l'angoisse de l'effacement de soi » (Anzieu \& Monjauze 23-24). 16. Sergueï Eisenstein, réal., Le Cuirassé Potemkine [БРОНЕНОсЕЦ ПотёмКИн], Goskino, 1925.

17. Pier Paolo Pasolini, réal., Théorème [Teorema], Aetos Produzioni Cinematografiche, 1969.

18. Bernardo Bertolucci, réal., Le Dernier Tango à Paris [Ultimo tango a Parigi], M.G.M., 1972.

19. «Voyez-vous, je pense que l'art rend compte, je pense que c'est un reportage. Et je pense que dans l'art abstrait, puisqu'il n'y a pas de reportage, il n'y a rien d'autre que l'esthétique du peintre et ses quelques sensations » (Bacon cité dans Leiris 52).

20. " a shorthand of sensation » (Hinton, 16'18").

21. La fluidité de la conscience est une construction de l'esprit. De fait, certaines pathologies neurologiques provoquent des phénomènes d'arrêts sur image dans l'esprit de ceux qui en sont atteints. Oliver Sacks évoque à ce propos le cas d'une de ses patientes qui, se faisant couler un bain, provoqua une inondation : «But she had been stuck, transfixed, at that perceptual moment when there was just an inch of water in the bath » (Sacks 4).

22. Jaubert, 11'30".

23. Dans le documentaire d'Adam Low, la sœur de l'artiste, Ianthe Knott explique que lors d'un voyage en Afrique du Sud, Francis avait été fasciné par le découpage du gibier abattu, notamment par la couleur de la chair des animaux que l'on dépeçait.

24. «The police photographs that you see, side face, full face " (Hinton, 43'45").

25. «A confrontation with the human presence » (Low, 59'52").

26. Jaubert, 18'25".

27. Hinton, 36'40".

28. Et Bacon de citer Wilde : « Yet each man kills the thing he loves » (Wilde 192) (Low, 57'05”).

\section{RÉSUMÉS}

Le thème de l'intempestivité comme manifestation soudaine, inattendue, parfois malvenue, s'inscrit au cœur de la pratique artistique de Francis Bacon, en partie fondée sur un contraste entre maîtrise et spontanéité, voire hasard. En découle une exploration de l'opposition entre empathie et nouveauté dérangeante émanant de certaines de ses œuvres, œuvres qui, par exemple, figurent souvent des cris de douleur comme étouffés d'un coup d'éponge. La question générale de la temporalité de l'œuvre d'art comme événement nous invite également à convoquer les notions d' "hétérochronie " et d' "image-fantôme"; mais aussi à explorer la fonction du triptyque comme commentaire méta-pictural, l'expression du mouvement dans la représentation fixe et la dimension épidermique de la toile. Enfin, nous abordons la question du rapport entre temps de la réception et temps de la représentation.

The notion of untimely art understood as a sudden, unpredictable and often unsought for phenomenon is at the heart of Bacon's artistic practice, which is partly based on a contrast between an overall feeling of mastery and sporadic, unanticipated outbursts of paint on the canvas. The screaming figures seem to beg for sympathy while the sponge, as if literally thrown on the painting, gags them unawares. Other notions correlated to that of time and temporality 
will be put under scrutiny, such as that of "heterochrony", and "ghost-images", with Bacon himself acknowledging that paintings by old masters haunted his cultural imagination. We will also examine how triptychs can be interpreted as meta-pictorial comments on the way vision works by dissecting space into various fragments, the metaphor of the canvas as an epidermal surface, and the twisted link that exists between the time it takes for the viewer to explore the canvas and the way time is represented within the frame.

\section{INDEX}

Mots-clés : épiderme, empathie, hétérochronie, image-fantôme, triptyque

Keywords : skin, sympathy, heterochrony, ghost-image, triptych

\section{AUTEURS}

\section{SÉVERINE LETALLEUR-SOMMER}

Séverine Letalleur-Sommer est maître de conférences à l'université Paris-Nanterre (Département d'Études anglophones - UFR Langues \& Cultures Etrangères). Ses travaux portent sur les théories des représentations, la sémiotique et la linguistique anglaise, et, plus précisément, sur la construction du sens visuel et linguistique et l'analyse croisée des discours théoriques dont ils font l'objet. Elle est membre du centre de recherche EA 370 CREA, Centre de Recherches Anglophones : http://anglais.u-paris10.fr/spip.php?article1085 